\title{
Calidad de la biopsia hepática en 10 instituciones hospitalarias de Bogotá
}

\section{Quality of liver biopsies taken in ten hospitals of Bogotá}

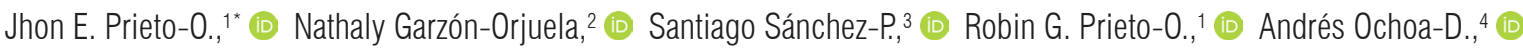
Javier Eslava-Schmalbach. ${ }^{2}$ []

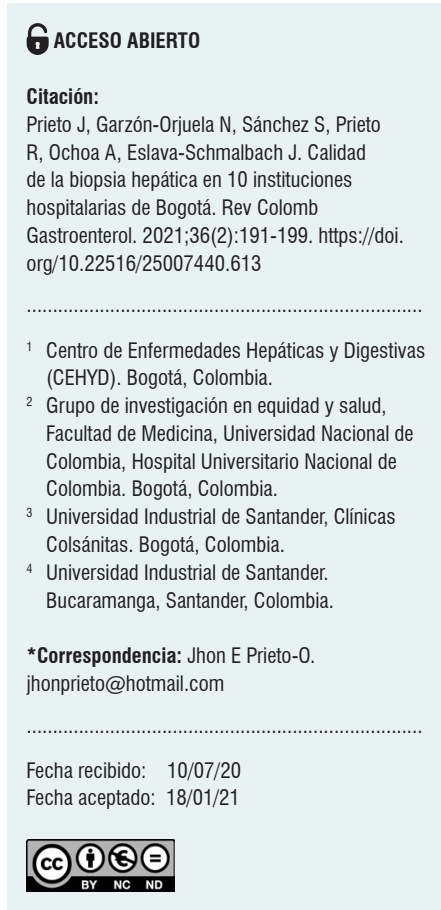

\begin{abstract}
Resumen
Introducción: la biopsia hepática es la prueba de oro para el diagnóstico de las enfermedades que comprometen el hígado, una muestra adecuada y una muy buena lectura son elementos que determinan la utilidad de la prueba y el impacto en la toma de decisiones. Objetivo: evaluar la calidad de las biopsias hepáticas a partir de la frecuencia de un diagnóstico definitivo en la lectura de las mismas y su relación con el número de espacios porta y su longitud informada. Materiales y métodos: estudio observacional retrospectivo basado en registros, entre el 1 de enero de 2010 y el 30 de julio de 2017. Se realizó la revisión de las historias clínicas de los pacientes a quienes se les realizó biopsia hepática y se evaluó el resultado de la patología. Resultados: se incluyeron 659 informes de patología de 10 instituciones. El porcentaje de reporte de espacios porta varió entre un $15 \%$ y un $87,4 \%$, entre las instituciones. La mediana de longitud de la biopsia fue 15 $\mathrm{mm}$ (rango intercuartílico [RIC]: 10-20) con el valor más bajo de 1,3 (1-1,5) y el más alto de 1,8 (1,4-2) y la del número de espacios porta fue de 10 (RIC: 7-15), con el valor más bajo de $5(1-8)$ y el más alto de $13(10-17)$. Los diagnósticos definitivos se presentaron entre $35 \%$ y $69 \%$, diagnósticos probables entre $25 \%$ y $63 \%$, y sin diagnóstico entre un $5 \%$ y 31,8\%. En el resultado de la regresión logística del diagnóstico y análisis univariado, se encontró que el número de espacios porta presentó un Odds ratio (OR) de 1,12 (intervalo de confianza [IC] 95 \%: 1,05-1,19) y la longitud, OR: 1,74 (1,06-2,87); con el análisis multivariado, el número de espacios porta sigue siendo significativo (OR: 1,12 [1,02 a 1,22], $p=0,011$ ). Conclusiones: En Bogotá existen 3 instituciones hospitalarias con adecuada calidad preanalítica en la toma de biopsias hepáticas y diagnósticos definitivos por encima del $60 \%$, asociados en esta serie con la presencia de un cilindro de tejido hepático de longitud y número de espacios porta adecuados. Con el análisis multivariado, el número de espacios porta presentó significancia. Se insiste en la importancia de la experiencia y entrenamiento del patólogo que evalúa la biopsia.
\end{abstract}

\section{Palabras clave}

Biopsia hepática, calidad de biopsia, diagnóstico definitivo.

\section{INTRODUCCIÓN}

Actualmente, el análisis del tejido hepático obtenido mediante una biopsia sigue siendo importante para establecer un diagnóstico, evaluar y estadificar la enfermedad subyacente, y dirigir el manejo en función de la histología informada (1); por tanto, la biopsia hepática sigue siendo una prueba fundamental en el estudio de las enfermeda- des hepáticas. Algunos la consideran la prueba de oro y otros, la mejor prueba existente; no obstante, la calidad de la prueba depende de varios factores: una indicación clínica adecuada, el médico que realiza el procedimiento (en nuestro medio, un radiólogo), la aguja que se usa, el número de punciones exitosas (cilindros de tejido obtenidos), la calidad de la muestra y el patólogo que la lee y la informa (2-5). 


\begin{abstract}
Introduction: Liver biopsy is the gold-standard test for the diagnosis of diseases involving the liver. An adequate sample and an accurate reading of the report are key to determine the usefulness of the test and its impact on decision-making. Objective: To assess the quality of liver biopsies based on the frequency of a "definitive diagnosis" in their report and their association with the number of portal spaces and reported length. Materials and methods: Record-based retrospective observational study, from January 1, 2010, to July 30, 2017. A review of the medical records of patients who underwent liver biopsy was performed, and the pathology result was evaluated. Results: 659 pathology reports from 10 hospitals were included. The percentage of portal space reporting varied between $15 \%$ and $87.4 \%$. The median biopsy length was $15 \mathrm{~mm}$ (IQR: 10-20) and the median number of portal spaces was 10 (IQR: 7-15). Definitive diagnoses were between $35 \%$ and $69 \%$, probable diagnoses between $25 \%$ and $63 \%$, and no diagnosis between $5 \%$ and $31.8 \%$. The logistic regression of the diagnosis and a univariate analysis found that the number of portal spaces had an OR of 1.12 (95\% Cl: $1.05-1.19)$, while length had an OR of $1.74(95 \% \mathrm{Cl}: 1.06-2.87)$. The multivariate analysis showed that the number of portal spaces is significant [OR: 1.12 (95\% Cl:1.02 to 1.22), $p=0.011$. Conclusions: In Bogotá, there are 3 hospitals with adequate pre-analytical quality of liver biopsies and definitive diagnoses above $60 \%$, which in this series is associated with the presence of a cylinder of liver tissue of adequate length and the number of portal spaces. Multivariate analysis showed that the number of portal spaces is significant. The importance of the experience and training of the pathologist who evaluates the biopsy is stressed.
\end{abstract}

Keywords

Liver biopsy; Quality of biopsy; Definitive diagnosis.

Guías previas sugieren cuáles son los aspectos de una buena muestra y recuerdan que una biopsia hepática entre 1 y $3 \mathrm{~cm}$ de longitud y entre 1,2 y $2 \mathrm{~mm}$ de diámetro representa 1/50 000 de la masa total del hígado (6). Se recomienda una muestra de al menos 2 a $3 \mathrm{~cm}$ de longitud, tomada con una aguja calibre 16 , que permita al patólogo evaluar 11 tractos portales completos, más el lobulillo hepático en una buena extensión, de tal forma que pueda emitir un diagnóstico definitivo o una sugerencia del mismo, teniendo en cuenta que el diagnóstico, la clasificación y la estadificación pueden ser incorrectos debido a un tamaño de muestra insuficiente. Si se sospecha cirrosis, se recomienda una aguja de corte en lugar de una aguja de succión, para obtener una mejor muestra y evitar la fragmentación de la misma $(1,7-10)$; se han formulado parámetros similares en las nuevas guías británicas (11).

La toma de la biopsia hepática se realiza en diferentes instituciones hospitalarias en la ciudad de Bogotá, Colombia, y se observan diferencias en los resultados finales; por tal motivo, el objetivo de este estudio fue evaluar la calidad de las biopsias hepáticas percutáneas a partir de la frecuencia de diagnóstico definitivo en la lectura de las mismas, en varias instituciones hospitalarias de Bogotá y su relación con el número de espacios porta y longitud de la biopsia en el informe final de patología.

\section{METODOLOGÍA}

Estudio observacional retrospectivo basado en registros de pacientes que asistieron a la consulta especializada de
Hepatología en el Centro de Enfermedades Hepáticas y Digestivas (CEHYD), en la ciudad de Bogotá. Se revisaron todas las historias clínicas de pacientes atendidos entre el 1 de enero del 2010 y el 30 de julio del 2017.

\section{Criterios de inclusión}

1. Antecedente de biopsia hepática percutánea en patología no tumoral.

2. Disponibilidad del resultado de patología original o su copia.

\section{Criterios de exclusión}

1. Biopsia hepática por cuña quirúrgica,

2. Biopsia de tumores hepáticos,

3. Muestra no apta para lectura según el patólogo,

4. No disponibilidad del resultado de patología,

5. Desconocimiento de la institución donde se realizó la biopsia.

Se realizó una evaluación del resultado de patología de la biopsia hepática y se consideraron las siguientes variables:

- El tamaño mencionado de la muestra: se tomó la longitud mayor en cm y se clasificó en 3 grupos de la siguiente manera: $<1 \mathrm{~cm}$, de $1-1,9 \mathrm{~cm} \mathrm{y} \geq 2 \mathrm{~cm}$. En caso de mención de varios fragmentos, se tomó en cuenta el de mayor tamaño.

- El número de espacios portales informados: se clasificó en 3 grupos: $<5$, de $6-10 y \geq 11$. 
- Información (sí o no) sobre las características del espacio porta.

- Información (sí o no) sobre las características del lobulillo hepático.

- Diagnóstico emitido: se consideró la probabilidad de certeza al analizar las conclusiones finales del resultado emitido por el patólogo, definido de la siguiente manera:

a. Diagnóstico definitivo, $100 \%$ de certeza: diagnóstico enunciado con claridad; el patólogo refiere "conclusivo de, ... altamente sugestivo", descripción histológica fuertemente sugestiva de una entidad.

b. Diagnóstico probable, $50 \%$ de certeza: "podría corresponder a...", "se sugiere...," "otros diagnósticos diferenciales".

c. No diagnóstico, $0 \%$ de certeza: no se enuncia o sugiere diagnóstico alguno. La descripción es incompleta.

Datos asociados con la biopsia (número de punciones, cilindros para lectura o fragmentación de la muestra) o con los patólogos (número por institución o su experiencia), no fueron tabulados por desconocimiento de los mismos.

El análisis del grado de fibrosis se llevó a cabo utilizando la escala METAVIR, si el informe era presentado con otra escala, se asimilaba a la METAVIR.

\section{ANÁLISIS ESTADÍSTICO}

La información recolectada fue resumida descriptivamente; las variables cualitativas se presentaron en frecuencias absolutas y las variables cuantitativas, mediante medidas de tendencia central y de dispersión, dependiendo de su distribución normal (prueba de Kolmogorov-Smirnov de normalidad). Debido a que los datos no presentaban una distribución normal, se muestra la diferencia entre instituciones de salud con la prueba Kruskal-Wallis. Se realizó un análisis univariado y multivariado con el tipo de diagnóstico (definitivo y no) y los valores de número de espacios porta y longitud de la biopsia mediante una regresión logística. Se utilizó la correlación Rho de Spearman. Todos los análisis fueron realizados en los programas estadísticos STATA versión 13 y R-4.0.3.

\section{RESULTADOS}

De un total de 927 pacientes que requirieron biopsia hepática durante el período analizado, 268 fueron excluidos por no cumplir con los criterios (especialmente debido a la no disponibilidad del informe final de patología y, en algunos casos, a historias clínicas incompletas). Finalmente, 659 pacientes fueron incluidos por contar con los informes de biopsias hepáticas, realizadas en 10 instituciones diferentes. El número de pacientes en cada institución fluctuó entre 14 y 314 . Del total de pacientes analizados, 601 informaron longitud de biopsia o espacios porta. El porcentaje de informe de espacios porta por institución varió entre un $15 \%$ y un $87,4 \%$ (Tabla 1). La gran mayoría de patólogos describió los espacios porta y los lobulillos hepáticos.

En toda la muestra, la mediana de longitud de la biopsia fue de $1,5 \mathrm{~cm}$ (rango intercuartílico [RIC]: 1-2) y la del número de espacios porta fue de 10 (RIC: 7-15). En las Figuras $\mathbf{1}$ y $\mathbf{2}$ se observan las diferencias significativas de longitud informadas por las diferentes instituciones. La mayoría de las instituciones obtuvo muestras de tejido hepático de mediana ( 1 a $1,9 \mathrm{~cm})$ o buena calidad $(\geq 2 \mathrm{~cm})$, como en el caso de la institución 4 , donde el $70 \%$ de las biopsias pertenecen a este último grupo. La distribución de los espacios porta por institución se muestra en la Figuras 3 y 4, y resaltan las instituciones 1, 2, 3 y 8 con biopsias que mostraron más de 11 espacios porta. Se aprecia una diferencia significativa en la proporción de diagnósticos definitivos, probables o no diagnósticos entre las instituciones. En la Figura 5 se muestra la proporción y el número de muestras procesadas por institución. No hubo correlación entre el volumen de muestras procesadas por institución y el número de espacios porta identificados o la longitud de la biopsia (Spearman Rho).

En cada institución los diagnósticos definitivos se presentaron entre $35 \%$ y $69 \%$; los diagnósticos probables, entre $25 \%$ y $63 \%$; y sin diagnóstico, entre $5 \%$ y $32 \%$. Un diagnóstico definitivo fue informado por 3 entidades en más del $60 \%$ de los casos, las otras 7 entidades lo informan en menos del $50 \%$. La entidad 9 tenía un bajo número de biopsias revisadas (solo 24), pero presentó el mayor porcentaje de pacientes con fibrosis cero. 62 pacientes, que corresponden al 9,4\% del total, presentaron cirrosis (F4) y, de ellos, el $77 \%$, no refería espacios porta en el informe final, con rangos entre el $58 \%$ y el $100 \%$.

En el resultado de la regresión logística del diagnóstico (definitivo y no), con el análisis univariado se encontró que el número de espacios presentó un Odds ratio (OR) de 1,12 (intervalo de confianza [IC] $95 \%$ : 1,05-1,19) y la longitud, un OR de $1,74(1,06-2,87)$; es decir que por cada aumento en un espacio porta se tiene 1,12 veces más probabilidad de presentar un diagnóstico definitivo y por cada aumento de $1 \mathrm{~cm}$ en la longitud de la biopsia se tiene 1,74 veces más probabilidad de presentar un diagnóstico definitivo. Con el análisis multivariado, el OR ajustado por sexo y obesidad para hacer diagnóstico definitivo sigue siendo significativo para el número de espacios porta (OR: 1,12 [1,02-1,22]). La longitud de la biopsia pierde su significancia (OR: $1,41[0,7-2,84])$ y las otras variables no son significativas (Tabla 2). 
Tabla 1. Características del resultado de la biopsia en las instituciones de salud $(\mathrm{n}=659)$

\begin{tabular}{|c|c|c|c|c|c|c|c|c|c|c|c|}
\hline & \multicolumn{11}{|c|}{ Instituciones de salud en Bogotá $(n=659)$} \\
\hline & $\begin{array}{c}1 \\
n=314\end{array}$ & $\begin{array}{c}2 \\
n=89\end{array}$ & $\begin{array}{c}3 \\
n=100\end{array}$ & $\begin{array}{c}4 \\
n=25\end{array}$ & $\begin{array}{c}5 \\
n=15\end{array}$ & $\begin{array}{c}6 \\
n=14\end{array}$ & $\begin{array}{c}7 \\
n=25\end{array}$ & $\begin{array}{c}8 \\
n=22\end{array}$ & $\begin{array}{c}9 \\
n=24\end{array}$ & $\begin{array}{c}10 \\
n=31\end{array}$ & $\begin{array}{c}\text { Total } \\
\mathrm{n}=659\end{array}$ \\
\hline Reporte del n. ${ }^{\circ}$ de espacios porta (\%) & 82,9 & 87,4 & 86 & 68 & 35,7 & 69,2 & 28 & 76,2 & 15 & 18,5 & 74,8 \\
\hline $\begin{array}{l}\text { Reporte de la descripción de espacios } \\
\text { porta (\%) }\end{array}$ & 93,9 & 97,7 & 93,4 & 91,7 & 92,9 & 92,9 & 87,5 & 100 & 85,7 & 77,8 & 93,2 \\
\hline \multirow{2}{*}{$\begin{array}{l}\text { Reporte de la descripción del lobulillo } \\
(\%)\end{array}$} & 88,7 & 91,9 & 91,7 & 91,7 & 100 & 92,9 & 95,7 & 100 & 95,2 & 77,8 & 90,5 \\
\hline & \multicolumn{11}{|c|}{ Diagnóstico (\%) } \\
\hline Definitivo & 69,4 & 60,2 & 45,5 & 41,7 & 35,7 & 42,8 & 36 & 61,9 & 4,6 & 35,7 & 56,8 \\
\hline Probable & 25,6 & 34,1 & 34,3 & 41,7 & 57,1 & 28,6 & 56 & 33,3 & 63,6 & 46,4 & 33,2 \\
\hline \multirow[t]{2}{*}{ No } & 5,0 & 5,7 & 20,2 & 16,6 & 7,2 & 28,6 & 8 & 4,8 & 31,8 & 17,9 & 10,0 \\
\hline & \multicolumn{11}{|c|}{ Grado de fibrosis (\%) } \\
\hline F0 & 30 & 48,2 & 27,1 & 41,7 & 28,6 & 46,1 & 19,0 & 38,1 & 60 & 32 & 33,8 \\
\hline F1 & 27,9 & 28,4 & 32,3 & 8,3 & 35,7 & 0 & 19,1 & 14,3 & 15 & 24 & 26,1 \\
\hline F2 & 13,2 & 5,8 & 17,7 & 25,0 & 7,1 & 15,4 & 19,0 & 28,6 & 5 & 20 & 14,0 \\
\hline F3 & 20,1 & 10,6 & 10,4 & 8,3 & 28,6 & 7,7 & 19,1 & 9,5 & 10 & 4 & 15,4 \\
\hline F4 & 8,7 & 7,1 & 12,5 & 16,7 & 0 & 30,8 & 23,8 & 9,5 & 10 & 20 & 10,7 \\
\hline $\begin{array}{l}\text { Total de F4 con espacios porta } \\
\text { reportados, } \mathrm{n}(\%)\end{array}$ & $5(21,7)$ & $1(16,6)$ & $5(41,6)$ & $1(25)$ & 0 & $1(33,3)$ & 0 & 0 & 0 & $1(20)$ & 14 \\
\hline $\begin{array}{l}\text { Total de F4 sin espacios porta } \\
\text { reportados, } n(\%)\end{array}$ & $18(78,2)$ & $5(83,3)$ & $7(58,3)$ & $3(75)$ & 0 & $2(66,6)$ & $5(100)$ & $2(100)$ & $2(100)$ & $4(80)$ & 48 \\
\hline Total de pacientes F4 por institución & 23 & 6 & 12 & 4 & 0 & 3 & 5 & 2 & 2 & 5 & 62 \\
\hline
\end{tabular}

Tabla 2. Análisis de regresión logística

\begin{tabular}{|lll|}
\hline \multicolumn{1}{c}{ Variable } & $\begin{array}{c}\text { Análisis univariado, } \\
\text { OR (IC) }\end{array}$ & $\begin{array}{r}\text { Análisis multivariado } \\
\text { (ajustado), } \\
\text { OR (IC 95 \%) }\end{array}$ \\
\hline $\begin{array}{l}\text { Cantidad de espacios } \\
\text { porta }\end{array}$ & $\begin{array}{l}1,12(1,05-1,19), \\
p=0,000\end{array}$ & $\begin{array}{l}1,12(1,02-1,22), \\
p=0,011\end{array}$ \\
\hline $\begin{array}{l}\text { Longitud de la biopsia } \\
\text { (cm) }\end{array}$ & $\begin{array}{l}1,74(1,06-2,87), \\
p=0,028\end{array}$ & $1,41(0,70-2,84)$ \\
\hline Obesidad & $1,13(0,56-2,27)$ & $1,62(0,52-5,05)$ \\
Sexo & $0,77(0,46-1,31)$ & $1,10(0,47-2,60)$ \\
\hline
\end{tabular}

\section{DISCUSIÓN}

Gracias a la biopsia hepática, el análisis histológico del hígado sigue siendo una prueba vigente en el estudio de los pacientes con enfermedades hepáticas $(1-5,11)$. Recientemente, Neuberger y colaboradores (11) han emitido unas recomendaciones acerca de las condiciones ideales de una biopsia hepática y varios artículos insisten en criterios que hacen que una biopsia hepática sea en conjunto adecuada y de buena calidad $(1,7-12)$; con base en lo anterior, se decidió evaluar la calidad de las biopsias hepáticas involucradas en el manejo de nuestros pacientes, mediante el análisis de 3 aspectos en el informe definitivo 


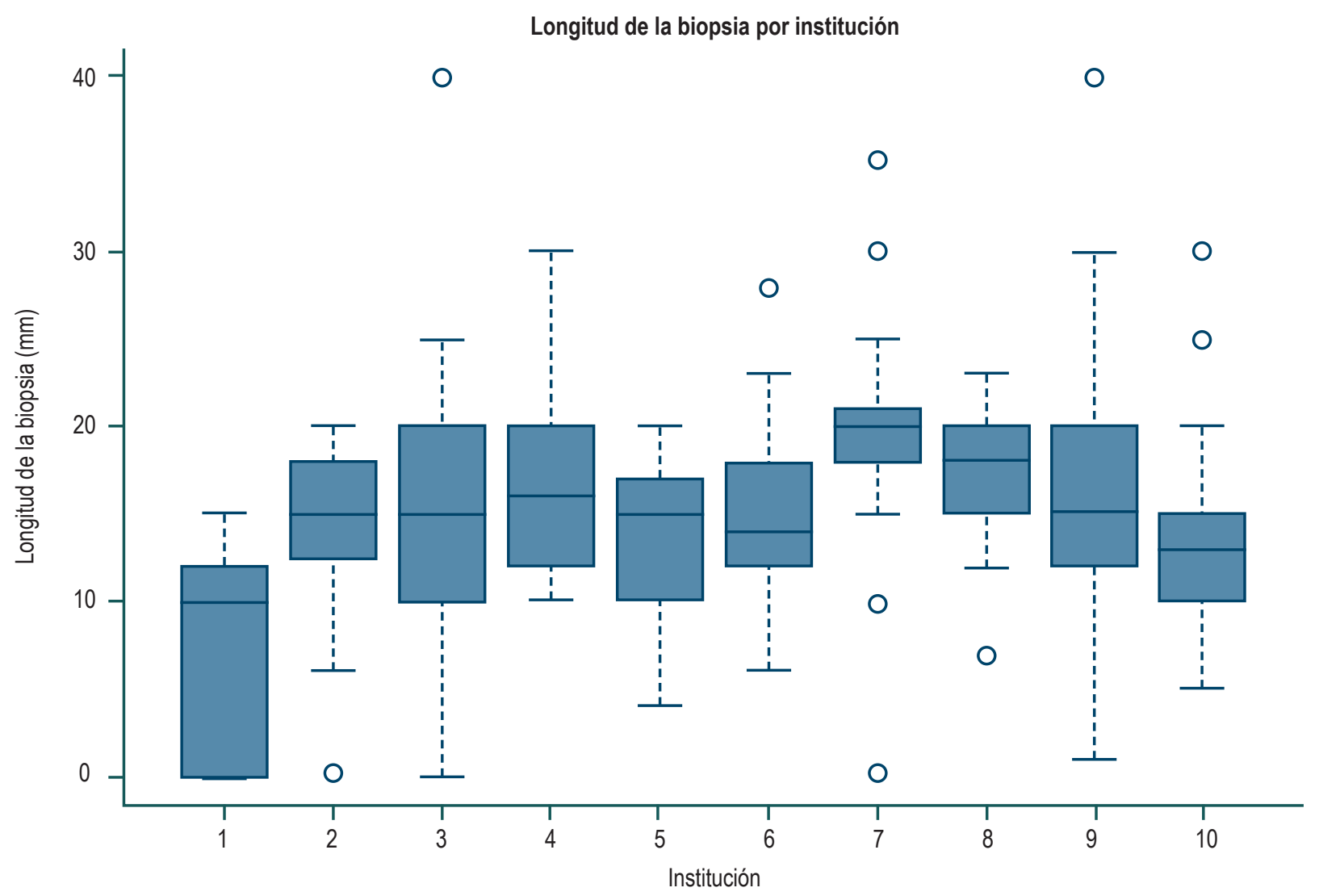

Figura 1. Longitud de la biopsia hepática por institución en mm. Kruskal-Wallis, $p=0,0001$ (prueba de Kruskal-Wallis).

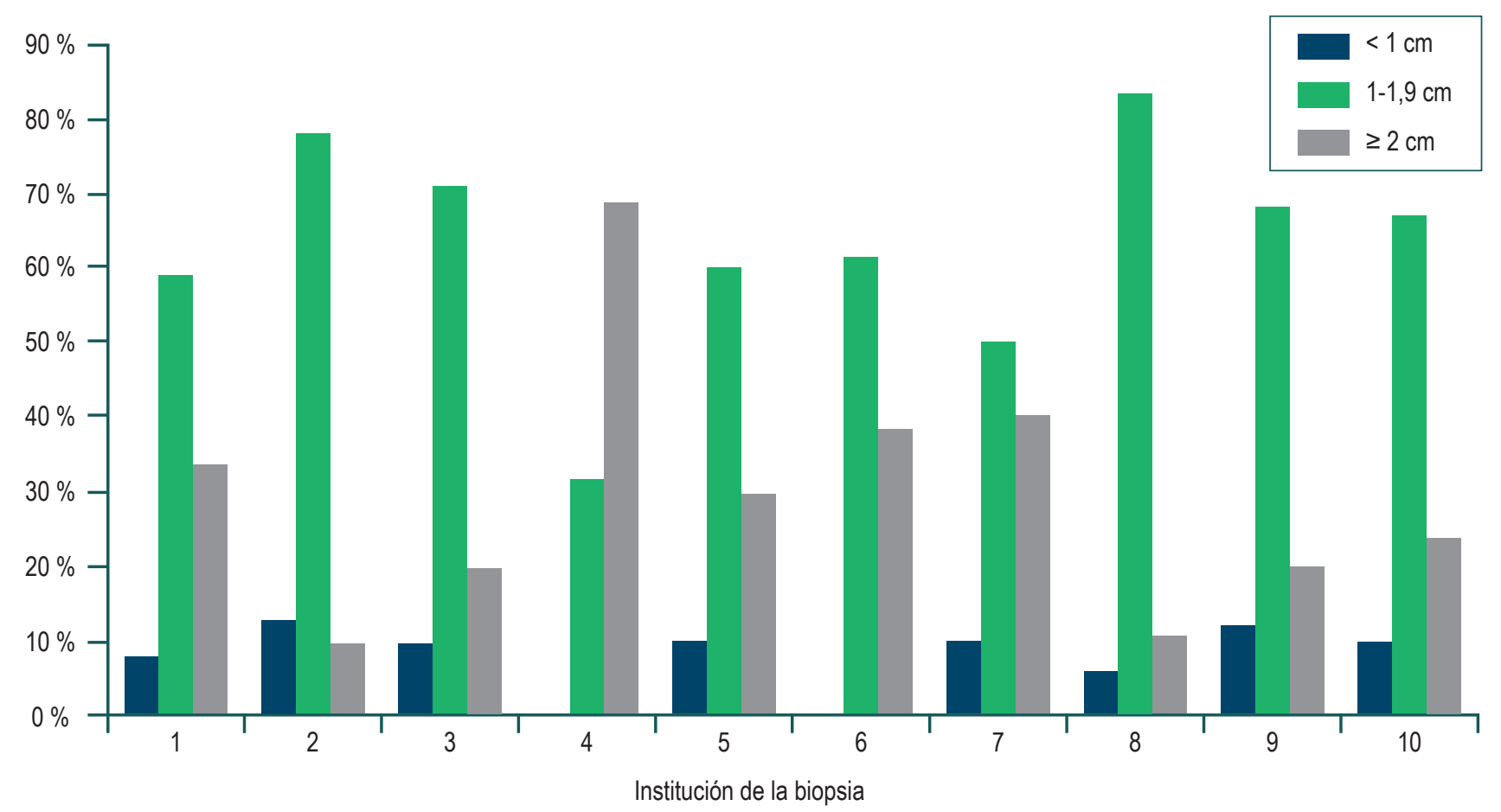

Figura 2. Distribución de las biopsias por tamaño y por institución. 


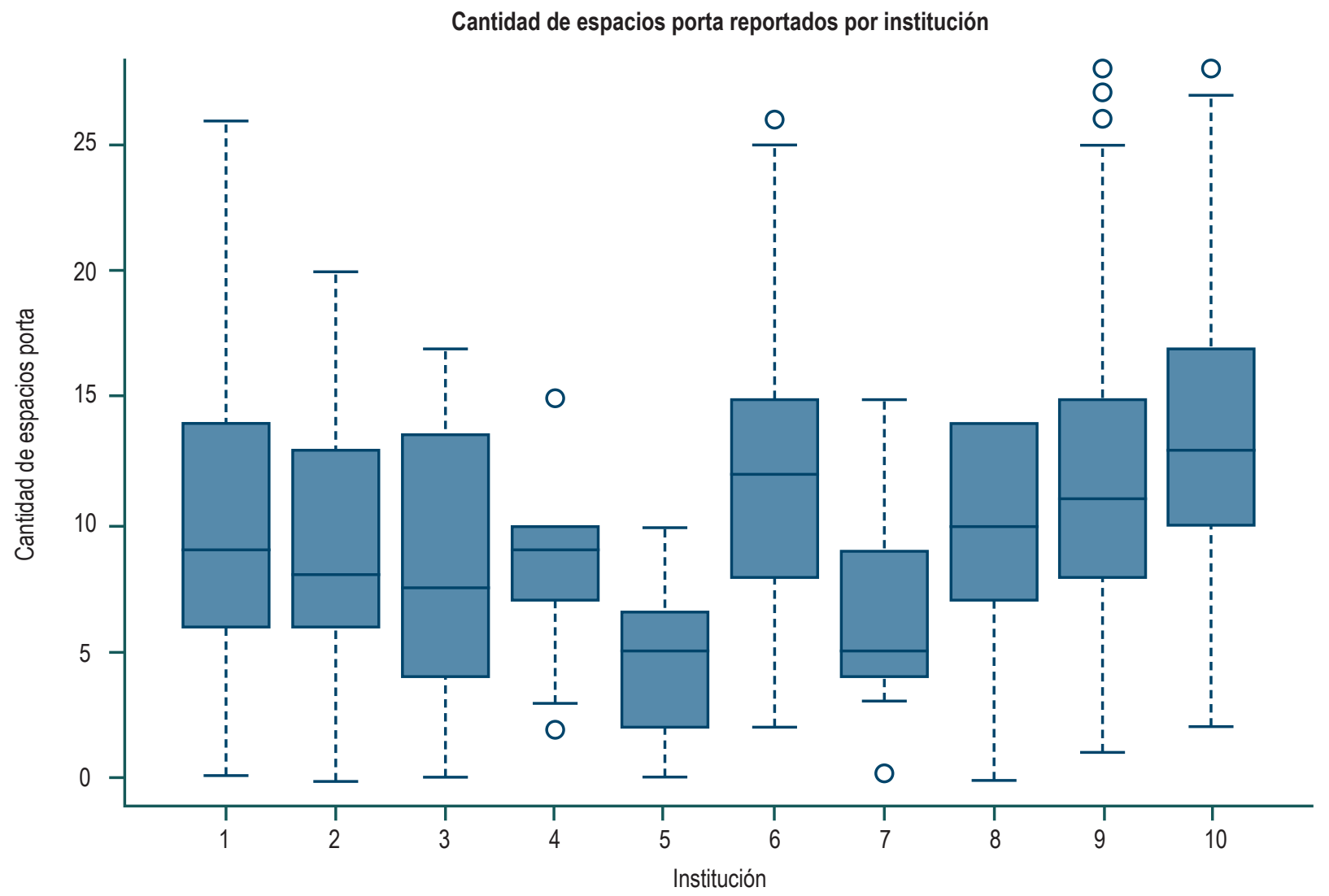

Figura 3. Cantidad de espacios porta por institución. Kruskal-Wallis, $p=0,0001$ (prueba de Kruskal-Wallis).

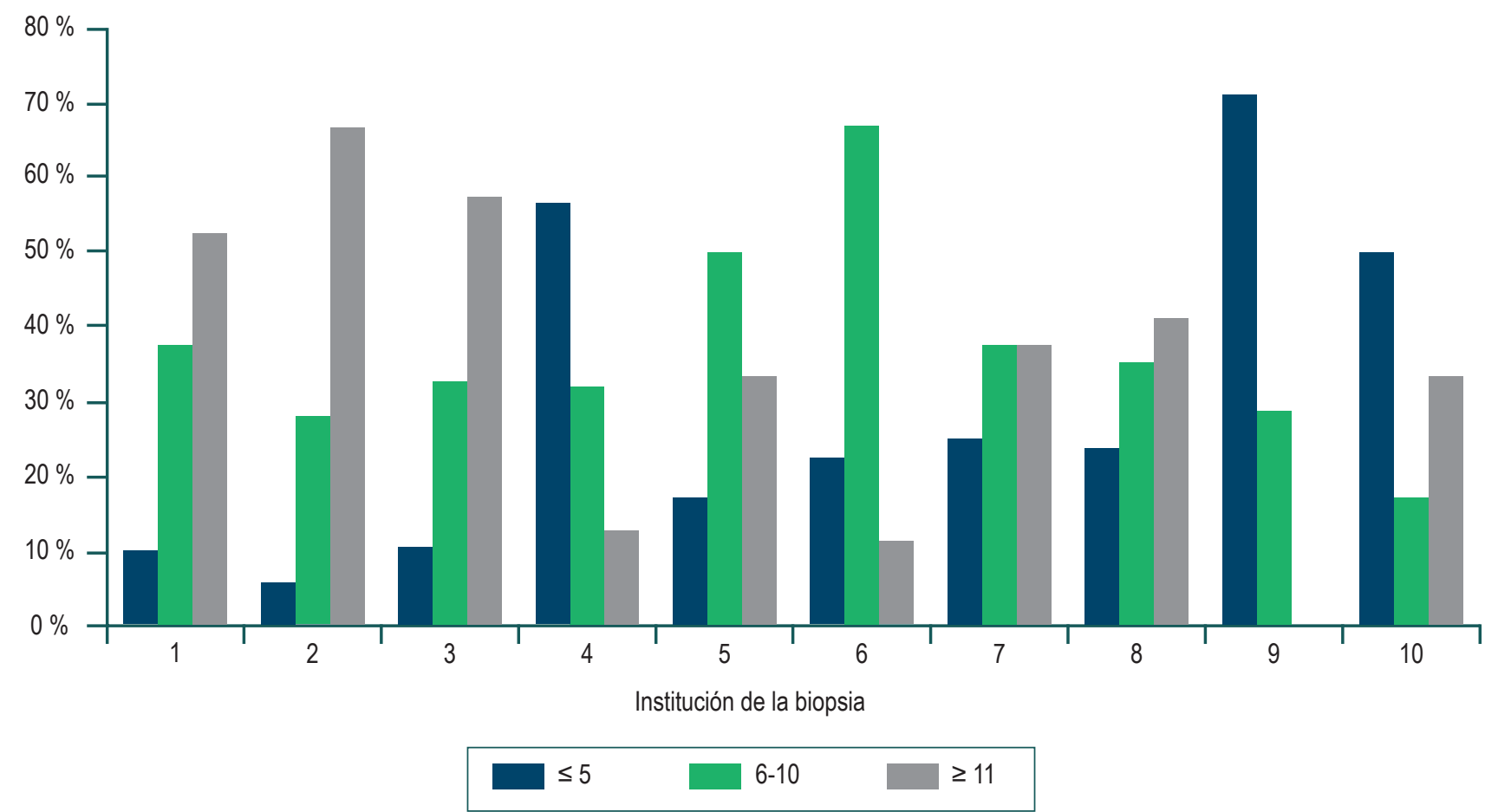

Figura 4. Distribución de los espacios porta según la institución y cantidad. 


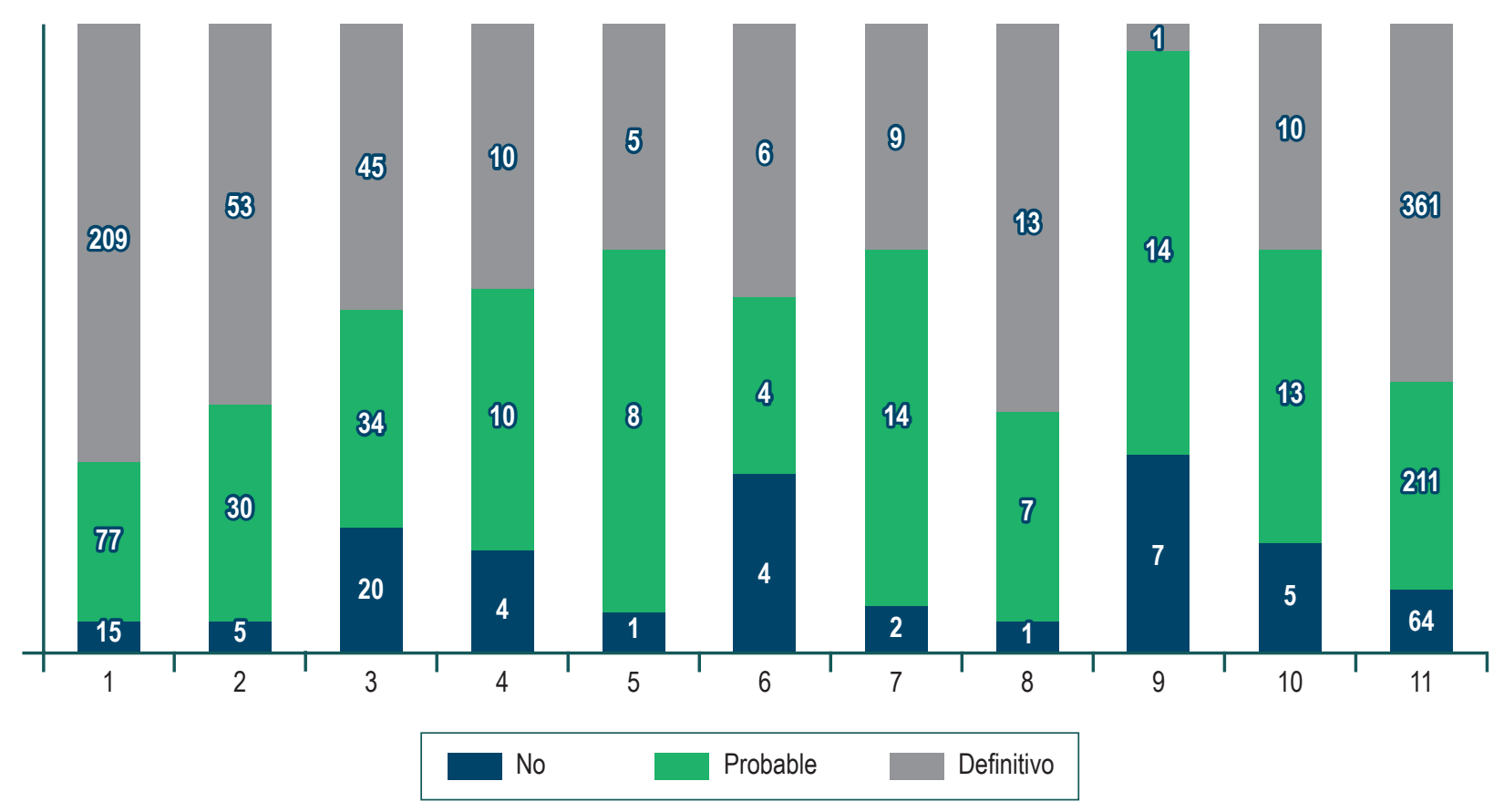

Figura 5. Tipo de diagnóstico por institución. Chi cuadrado $\left(\chi^{2}\right), p<0,001$.

del patólogo: longitud de la biopsia, número de espacios porta y presencia definitiva, parcial o no de un diagnóstico, todos ellos elementos cruciales en el informe del patólogo.

El tamaño ideal de una biopsia hepática ha variado (12). En hepatitis $\mathrm{C}$ crónica, inicialmente se sugería que una biopsia de 1-1,5 cm de longitud con 4-6 espacios porta era suficiente para la estadificación $(7,13,14)$; mientras que otros autores mencionan un tamaño mínimo de 2 a $2,5 \mathrm{~cm}$ como el ideal, con al menos 11 espacios porta (10, 15-17), teniendo en cuenta que, a mayor tamaño, mayor número de espacios porta (17). Estas últimas recomendaciones son las sugeridas en la actualidad $(1,5,10,11,14)$. En nuestro estudio, las medianas de longitud y espacios porta fueron respectivamente de $1,5 \mathrm{~cm}$ y 10 , en los límites inferiores de una biopsia de buena calidad, e informan el número de espacios porta disponibles para lectura en un $74 \%$ de las muestras. Con respecto a las descripciones disponibles de los espacios porta, el porcentaje total es superior al $90 \%$.

Es de resaltar que 4 instituciones con bajo número de pacientes informaron el número de espacios porta en menos de un $40 \%$ de los casos y, por el contrario, la institución 8 , con similar número de pacientes, informó espacios porta en el $76 \%$; esto podría explicarse, en parte, por la presencia de cirrosis. De hecho, un total de 62 pacientes $(9,4 \%$ del total de no informes de espacios porta) fue clasificado como F4 y explica algunos de estos casos. Por otra parte, podría tratarse de poca experiencia o entrenamiento de los patólogos de las primeras 3 instituciones, y gran experiencia o entrenamiento del patólogo de la institución número 8 .

El calibre de la aguja y el número de punciones exitosas (o cilindros de tejido hepático obtenidos por el radiólogo) tienen relación directa con el tamaño de la biopsia y el número de espacios porta que el patólogo revisa; por tanto, se recomienda un aguja calibre 16 y al menos 3 muestras no fragmentadas de tamaño suficiente $(18,19)$. Aunque un estudio que compara 2 contra 3 punciones muestra mayor riesgo al realizar 3 tomas, dado fundamentalmente por dolor en el $74 \%$ y hemorragia en el $33 \%$ de los casos (20), en otros estudios (uno con más de 15000 biopsias) no se demostró este riesgo $(21,22)$. En nuestro estudio no tuvimos acceso a estos datos, pero lo que se observó fue cierta tendencia en nuestro medio a usar agujas más delgadas y usualmente con una sola punción (cilindro), posiblemente por el temor de las complicaciones.

La experiencia del patólogo o su entrenamiento en enfermedades hepáticas es un aspecto fundamental en la calidad del informe de la biopsia hepática y en algunas ocasiones puede suplir los déficits de una muestra. En el caso opuesto, algunos estudios refieren errores de diagnóstico (clínicamente significativos) por patólogos sin experiencia en más del $25 \%$ de los casos en una institución universitaria (23, 24), por lo cual, ante la duda diagnóstica, se recomienda un 
segundo concepto $(24,25)$. En nuestra serie, este aspecto inherente a una buena experticia del patólogo podría explicar los hallazgos ya mencionados de la institución 8 y, por el contrario, la carencia de esta explicaría hallazgos como el bajo diagnóstico definitivo en varias instituciones con buen número de pacientes, como la institución 3 , o con muestras relativamente buenas, como las instituciones 5 y 6 , donde las medianas de las muestras fueron $\geq 1,6 \mathrm{~cm}$ y $\geq 9$ espacios porta; adicionalmente, también explicaría la no descripción de espacios porta y de lobulillos en algunas instituciones.

Se encontró que 3 entidades, la 1,2 y 8 , informaron diagnósticos definitivos en más del $60 \%$ de los casos, las 2 primeras entidades tenían biopsias cercanas a lo ideal en cuanto a tamaño y número de espacios porta; y la tercera institución curiosamente tenía un bajo número de pacientes y biopsias de mediana calidad, situación en la que nuevamente y con seguridad juega un papel fundamental la experiencia o entrenamiento del patólogo.

En relación con el estadiaje del grado de fibrosis, la institución 9 , con un bajo número de biopsias realizadas y el menor porcentaje de informe de número de espacios porta y diagnóstico definitivo, presentó el mayor porcentaje de pacientes con fibrosis cero; este hallazgo se describe en la literatura y se sabe que, a menor calidad de la muestra, mayores probabilidades de estadiajes bajos (10, 15 ). Igualmente, una mala muestra genera una mayor probabilidad de errores de muestreo debido a la heterogeneidad del hígado en las diferentes etapas de la enfermedad, error que se acrecienta con el mayor grado de fibrosis (4, 26-28). En este mismo sentido, es importante resaltar que el paciente con cirrosis tiene una alta probabilidad de fragmentación de la biopsia, hallazgo demostrado por Poynard, quien considera que "la fragmentación es un signo de cirrosis, independientemente de la longitud de la biopsia" (29).

Reconocemos las limitaciones del estudio, como su carácter retrospectivo y centrado en el análisis del informe de patología, lo cual limita el análisis de otras variables; sin embargo, los resultados sugieren que una adecuada muestra y una lectura de un médico patólogo experto, basado seguramente también en una información clínica del paciente, hacen que el diagnóstico emitido sea mucho más confiable.

\section{CONCLUSIONES}

En nuestro estudio se encontraron 3 instituciones hospitalarias de Bogotá con una adecuada calidad preanalítica en la toma de las biopsias y diagnósticos categóricos por encima del $60 \%$ que orientan al clínico. El diagnóstico definitivo en la biopsia hepática se asoció en esta serie con la presencia de un cilindro de tejido hepático de longitud y número de espacios porta adecuados. Con el análisis multivariado, el número de espacios porta presentó significancia. Se insiste en la importancia de la experiencia y entrenamiento del patólogo que evalúa la biopsia y, posiblemente, en el número de pacientes atendidos por institución (lo que a su vez implicaría mayor experiencia) como elementos coadyuvantes en estos resultados, aspectos que no fueron evaluados.

\section{Conflictos de interés}

Los autores declaran no tener conflictos de interés.

\section{Fuentes de financiación}

Con recursos propios.

\section{REFERENCIAS}

1. Rockey DC, Caldwell SH, Goodman ZD, Nelson RC, Smith AD; American Association for the Study of Liver Diseases. Liver biopsy. Hepatology. 2009;49(3):1017-44. https://doi.org/10.1002/hep.22742

2. Bedossa P, Carrat F. Liver biopsy: the best, not the gold standard. J Hepatol. 2009;50(1):1-3. https://doi.org/10.1016/j.jhep.2008.10.014

3. Poynard T, Benhamou Y, Thabut D, Ratziu V. Liver biopsy: the best standard...when everything else fails. J Hepatol. 2009;50(6):1267-8. https://doi.org/10.1016/j.jhep.2009.02.010

4. Tapper EB, Lok ASF. Use of Liver Imaging and Biopsy in Clinical Practice. N Engl J Med. 2017;377(8):756-768. https://doi.org/10.1056/NEJMra1610570
5. Khalifa A, Rockey DC. The utility of liver biopsy in 2020 . Curr Opin Gastroenterol. 2020;36(3):184-191. https://doi.org/10.1097/MOG.0000000000000621

6. Klein, M.A. Diagnostic Liver Pathology. By R.G. Lee, 517 pp. St. Louis: Mosby, 1994. Hepatology, 20: 1645-1646. https://doi.org/10.1002/hep.1840200644

7. Bravo AA, Sheth SG, Chopra S. Liver biopsy. N Engl J Med. 2001;344(7):495-500. https://doi.org/10.1056/NEJM200102153440706

8. Schiano TD, Azeem S, Bodian CA, Bodenheimer HC Jr, Merati S, Thung SN, et al. Importance of specimen size in accurate needle liver biopsy evaluation of patients with chronic hepatitis C. Clin Gastroenterol Hepatol. 
2005;3(9):930-5.

https://doi.org/10.1016/s1542-3565(05)00541-0

9. Cholongitas E, Quaglia A, Samonakis D, Senzolo M, Triantos C, Patch D, et al. Transjugular liver biopsy: how good is it for accurate histological interpretation? Gut. 2006;55(12):1789-94. https://doi.org/10.1136/gut.2005.090415

10. Colloredo G, Guido M, Sonzogni A, Leandro G. Impact of liver biopsy size on histological evaluation of chronic viral hepatitis: the smaller the sample, the milder the disease. J Hepatol. 2003;39(2):239-44. https://doi.org/10.1016/s0168-8278(03)00191-0

11. Neuberger J, Patel J, Caldwell H, Davies S, Hebditch V, Hollywood C, et al. Guidelines on the use of liver biopsy in clinical practice from the British Society of Gastroenterology, the Royal College of Radiologists and the Royal College of Pathology. Gut. 2020;69(8):1382-1403. https://doi.org/10.1136/gutjnl-2020-321299

12. Cholongitas E, Senzolo M, Standish R, Marelli L, Quaglia A, Patch D, et al. A systematic review of the quality of liver biopsy specimens. Am J Clin Pathol. 2006;125(5):710-21. https://doi.org/10.1309/W3XC-NT4H-KFBN-2G0B

13. Ryder SD, Irving WL, Jones DA, Neal KR, Underwood JC; Trent Hepatitis C Study Group. Progression of hepatic fibrosis in patients with hepatitis C: a prospective repeat liver biopsy study. Gut. 2004;53(3):451-5. https://doi.org/10.1136/gut.2003.021691

14. Coral GP, Antunes AD, Serafini AP, Araujo FB, Mattos AA. Liver biopsy: importance of specimen size in the diagnosis and staging of chronic viral hepatitis. Rev Inst Med Trop Sao Paulo. 2016;58:10. https://doi.org/10.1590/S1678-9946201658010

15. Bedossa $P$, Dargère $D$, Paradis V. Sampling variability of liver fibrosis in chronic hepatitis C. Hepatology. 2003;38(6):1449-57. https://doi.org/10.1016/j.hep.2003.09.022

16. Crawford AR, Lin XZ, Crawford JM. The normal adult human liver biopsy: a quantitative reference standard. Hepatology. 1998;28(2):323-31. https://doi.org/10.1002/hep.510280206

17. Röcken C, Meier H, Klauck S, Wolff S, Malfertheiner P, Roessner A. Large-needle biopsy versus thin-needle biopsy in diagnostic pathology of liver diseases. Liver. 2001;21(6):391-7. https://doi.org/10.1034/j.1600-0676.2001.210605.x

18. Cholongitas E, Quaglia A, Samonakis D, Mela M, Patch D, Dhillon AP, et al. Transjugular liver biopsy in patients with diffuse liver disease: comparison of three cores with one or two cores for accurate histological interpretation. Liver Int. 2007;27(5):646-53. https://doi.org/10.1111/j.1478-3231.2007.01496.x

19. Sporea I, Gherhardt D, Popescu A, Sirli R, Cornianu M, Herman D, et al. Does the size of the needle influence the number of portal tracts obtained through percutaneous liver biopsy? Ann Hepatol. 2012;11(5):691-5. https://doi.org/10.1016/S1665-2681(19)31444-9

20. Chi H, Hansen BE, Tang WY, Schouten JN, Sprengers D, Taimr P, et al. Multiple biopsy passes and the risk of complications of percutaneous liver biopsy. Eur J Gastroenterol Hepatol. 2017;29(1):36-41. https://doi.org/10.1097/MEG.0000000000000731

21. Midia M, Odedra D, Shuster A, Midia R, Muir J. Predictors of bleeding complications following percutaneous imageguided liver biopsy: a scoping review. Diagn Interv Radiol. 2019;25(1):71-80. https://doi.org/10.5152/dir.2018.17525

22. Atwell TD, Smith RL, Hesley GK, Callstrom MR, Schleck CD, Harmsen WS, et al. Incidence of bleeding after 15,181 percutaneous biopsies and the role of aspirin. AJR Am J Roentgenol. 2010;194(3):784-9. https://doi.org/10.2214/AJR.08.2122

23. Bejarano PA, Koehler A, Sherman KE. Second opinion pathology in liver biopsy interpretation. Am J Gastroenterol. 2001;96(11):3158-64. https://doi.org/10.1111/j.1572-0241.2001.05273.x

24. Hahm GK, Niemann TH, Lucas JG, Frankel WL. The value of second opinion in gastrointestinal and liver pathology. Arch Pathol Lab Med. 2001;125(6):736-9. https://doi.org/10.1043/00039985(2001)125<0736:TVOSOI>2.0.CO;2

25. Tomaszewski JE, Bear HD, Connally JA, Epstein JI, Feldman M, Foucar K, et al. Consensus conference on second opinions in diagnostic anatomic pathology. Who, What, and When. Am J Clin Pathol. 2000;114(3):329-35. https://doi.org/10.1093/ajcp/114.3.329

26. Ratziu V, Charlotte F, Heurtier A, Gombert S, Giral P, Bruckert E, et al. Sampling variability of liver biopsy in nonalcoholic fatty liver disease. Gastroenterology. 2005;128(7):1898-906. https://doi.org/10.1053/j.gastro.2005.03.084

27. Regev A, Berho M, Jeffers LJ, Milikowski C, Molina EG, Pyrsopoulos NT, et al. Sampling error and intraobserver variation in liver biopsy in patients with chronic $\mathrm{HCV}$ infection. Am J Gastroenterol. 2002;97(10):2614-8. https://doi.org/10.1111/j.1572-0241.2002.06038.x

28. Soloway RD, Baggenstoss AH, Schoenfield LJ, Summerskill WH. Observer error and sampling variability tested in evaluation of hepatitis and cirrhosis by liver biopsy. Am J Dig Dis. 1971;16(12):1082-6. https://doi.org/10.1007/BF02235164

29. Poynard T, Halfon P, Castera L, Charlotte F, Le Bail B, Munteanu M, et al. Variability of the area under the receiver operating characteristic curves in the diagnostic evaluation of liver fibrosis markers: impact of biopsy length and fragmentation. Aliment Pharmacol Ther. 2007;25(6):733-9. https://doi.org/10.1111/j.1365-2036.2007.03252.x 